\title{
Effects of Platycodin D on Reflux Esophagitis due to Modulation of Antioxidant Defense Systems
}

\author{
Su-Yeon Cho, ${ }^{1}$ Chang-Hyun Song, ${ }^{2,3}$ Ji-Eun Lee, $^{2,3}$ Seong Hun Choi, ${ }^{2}$ \\ Sae-Kwang Ku $\left(\mathbb{0},{ }^{2,3}\right.$ and Soo-Jin Park $\mathbb{C}^{2,3}$ \\ ${ }^{1}$ Medical Products Safety Division, Daejeon Regional Food and Drug Administration, Ministry of Food and Drug Safety, \\ Daejeon, Republic of Korea \\ ${ }^{2}$ Department of Histology and Anatomy, College of Korean Medicine, Daegu Haany University, Gyeongsan, Republic of Korea \\ ${ }^{3}$ The Medical Research Center for Globalization of Herbal Formulation, Daegu Haany University, Gyeongsan, Republic of Korea
}

Correspondence should be addressed to Sae-Kwang Ku; gucci200@hanmail.net and Soo-Jin Park; sjp124@gmail.com

Received 29 December 2017; Revised 11 February 2018; Accepted 21 February 2018; Published 26 March 2018

Academic Editor: Yuan Xu

Copyright (c) $2018 \mathrm{Su}$-Yeon Cho et al. This is an open access article distributed under the Creative Commons Attribution License, which permits unrestricted use, distribution, and reproduction in any medium, provided the original work is properly cited.

\begin{abstract}
Aims. The effects of platycodin D (PD) pretreatment were examined in reflux esophagitis (RE) induced rats. Methods. Sham, control, and omeprazole (OMP) group were pretreated with distilled water or OMP as a reference, respectively, and PD pretreated groups were given 3 different PD doses once a day for 7 days. One hour after last pretreatment, RE was induced by ligation of the forestomach and pylorus. At $8 \mathrm{~h}$ after operation, all animals were sacrificed. Results. PD showed significant dose-dependent reduction of gastric secretion, myeloperoxidase activity, and RE lesion areas of esophagus and stomach mucosa. There was a reduction of lipid peroxidation in 2 doses of PD groups and elevation of antioxidant enzyme activity in all PD groups. Gastric hexose and sialic acid were significantly increased in PD groups, while collagen was reduced. Plasma histamine levels were significantly reduced in all PD groups, but not in the OMP group. Total invasive lesion sizes of esophagus and gastric fundus were significantly decreased in all PD groups. Thicknesses in esophagus of all PD groups were significantly decreased and thicknesses of funds were significantly increased except lowest PD dose. Conclusions. Therapeutic effects of PD on the esophageal and gastric lesions were shown in RE induced rats dose-dependently. The PD pretreatment had significant antioxidant effects with regulation of histamine levels. This study provides useful information regarding the effectiveness of the drug for RE and further novel drug discovery using natural herbal products.
\end{abstract}

\section{Introduction}

Reflux esophagitis (RE) is an esophageal mucosal injury and is caused by reduced motility in the lower esophageal sphincter, leading to an abnormal increase in gastric contents [1]. The abnormal reflux of gastric contents typically damages the distal esophagus and gastroesophageal junction, inducing inflammatory responses in the esophageal mucosa. The general RE symptoms are regurgitation, dysphagia, and heartburn, but chronic RE can induce severe complications including laryngitis, esophageal stricture, and Barrett's esophagus and may eventually lead to cancer [2]. RE is a common digestive tract disorder in Western countries with a relatively high prevalence of $10-30 \%$ of individuals reporting weekly symptoms [3] and is also increasingly common in Korea, probably due to irregularities in lifestyle, obesity, and dietinduced gastric irritation by alcohol, caffeine, or aspirin [4]. Recent studies have shown a strong connection between the pathogenesis of RE and oxygen-derived free radicals and that oxidative stress has important roles in RE pathogenesis $[5,6]$.

Current $\mathrm{RE}$ treatments can be categorized according to the following mechanisms: antacids, $\mathrm{H}_{2}$ receptor antagonists, proton pump inhibitors (PPI), and gastric motility agents. Although many drugs have been developed to treat RE, more than $40-60 \%$ of patients still suffer from stricture or other complications including cancers and many remain far from full recovery despite adequate treatment time with acid secretion suppressants [7]. Therefore, effective therapeutic strategies remain in the public interest, thereby underlining the need for further drug discovery. 
Attention towards natural products as novel drug materials has been increasing in the drug development area. Platycodon grandiflorum, also known as platycodi radix, are commonplace plants in Korea, Japan, and China, and the root is used as a food and herbal medicine for preventing flu, cough, metabolic disorders, stomachache, diarrhea, postnatal illness, insomnia, and infection. In particular, the root is known to contain a large quantity of saponin, which is known to consist of platycodin $\mathrm{A}, \mathrm{C}$, and $\mathrm{D}$, as well as polygalacin $\mathrm{D}$, spinasterol, spinasterol glucoside, inulin, and so on $[8,9]$. Platycodin D (PD) is a major medicinal element of Platycodon grandiflorum [9] and has also been shown to have anticancer $[10,11]$, anti-inflammatory $[12,13]$, antiobesity [14], antidiabetes [15], cholesterol-lowering [16], and antinociceptive [17] properties as well as immunoregulatory effects [18]. PD also affords protection against oxidative stress which is useful in treating various diseases $[19,20]$. Therefore, the therapeutic effects of PD on esophageal and stomach mucosal injuries were examined in RE induced rats in this study.

\section{Materials and Methods}

2.1. Animals. All experiments were conducted after attaining approval of protocol by Institutional Animal Care and Use Committee (IACUC) at Daegu Haany University (Gyeongsan, Korea; IACUC number DHU013-097, December 24, 2013). Male Sprague-Dawley rats (6 weeks, Japan SLC Inc, Shizuoka, Japan) were maintained at $20-25^{\circ} \mathrm{C}$ and humidity of $40-45 \%$ with $12 \mathrm{~h}$ light/dark cycle. Water and normal rodent pellet diet were supplied ad libitum.

2.2. Materials Preparation and Pretreatments. PD, a bidesmoside triterpenoid consisting of an aglycone moiety, 3-Glc, and 28-O-Api-Xyl-Rha-Ara, was extracted from raw platycodin radix by previous method [16] and provided from Glucan Corp. Ltd (Busan, Korea). The PD was identified by comparing to confirmed PD sample based on radio frequency (Rf), fast atom bombardment mass spectrometry (FAB-MS; $=1225.38)$, and $\left[{ }^{13} \mathrm{C}\right]-\mathrm{NMR}$ spectra. The purified PD was in light yellow powder form and stored in a desiccator at $4{ }^{\circ} \mathrm{C}$ until use. PD was dissolved to at least $20 \mathrm{mg} / \mathrm{ml}$ in distilled water and used at 3 different doses $(200,100$, or $50 \mathrm{mg} / \mathrm{kg})$. Omeprazole (OMP) (Sigma, MO, USA) was dissolved in distilled water and used at a dose of $10 \mathrm{mg} / \mathrm{kg}$ as a reference drug [21]. The OMP was kept in a desiccator at room temperature before use. After acclimatization for 1 week, rats were allocated into six groups of 8 rats with random manner. OMP, PD 200, PD 100, and PD 50 groups were pretreated with omeprazole $10 \mathrm{mg} / \mathrm{kg}$, PD $200 \mathrm{mg} / \mathrm{kg}, 100 \mathrm{mg} / \mathrm{kg}$, and $50 \mathrm{mg} / \mathrm{kg}$ once a day for a week, respectively. Sham and control group were orally administered distilled water once a day for a week.

2.3. Induction of RE and Sampling. Sham and the other groups received a sham surgery or RE induction surgery $1 \mathrm{~h}$ after the last pretreatment, respectively [22]. Rats were anesthetized with Zoletil mixture ${ }^{\mathrm{TM}}(25 \mathrm{mg} / \mathrm{kg}$, i.p.; Virbac, France). For RE induction, the abdomen was opened by a $2 \mathrm{~cm}$ median incision. The transitional region between the

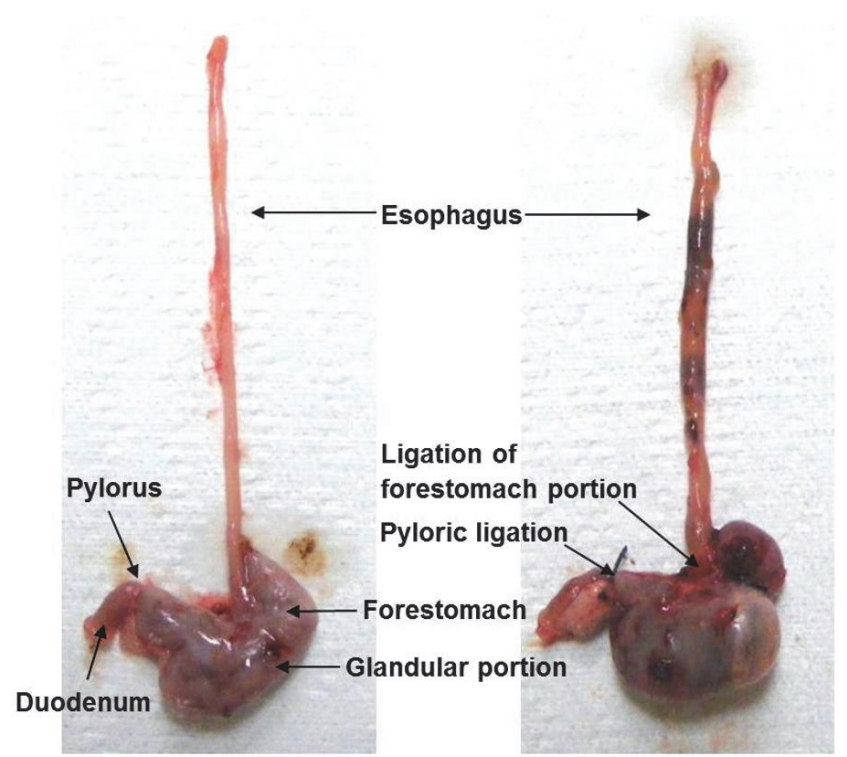

(a)

(b)

FIGURE 1: Sham surgery (a) or reflux esophagitis induction surgery by ligation of the forestomach and pylorus (b).

forestomach and corpus and the pylorus portion were ligated with a 2-0 silk thread (B. Braun Surgical SA, Spain) (Figure 1). A $1 \mathrm{~cm}$ longitudinal cardiomyotomy was performed across the gastroesophageal junction. The incised region was immediately sutured. For the sham, the abdomen was opened in the same manner as the RE induction and closed immediately without ligation and cardiomyotomy. All animals were sacrificed after collection of $1 \mathrm{ml}$ of blood from the orbital plexus at $8 \mathrm{~h}$ after surgery and samples of esophagus and stomach tissue; gastric contents were collected.

2.4. Measurements of Body Weights Changes. Body weight was measured 1 day before pretreatment and at days 0,1 , 5 , and 6 after pretreatment using an automated electronic balance (Precisa Instrument, Switzerland). Body weight gains were calculated according to the following equation:

$$
\begin{aligned}
& \text { Body weight changes }(\mathrm{g}) \\
& =\text { body weight at sacrifice }(\text { day } 6) \\
& \quad \text { - body weight at initial pretreatment (day } 0) \text {. }
\end{aligned}
$$

2.5. Measurements of Gross Lesions. Tissue samples of esophagus and stomach were fixed in $10 \%$ neutral buffered formalin. Gross lesions of esophagus or stomach were measured as a total area $\left(\mathrm{mm}^{2}\right)$ with a light microscope using a $4 \mathrm{x}$ objective lens (Nikon, Japan) and scored according to previous method [22].

2.6. Measurements of Gastric Volume and Contents. The gastric contents were centrifuged for $5 \mathrm{~min}$ at $2000 \times \mathrm{g}$, and the volume of the supernatant was described as $\mathrm{ml} / \mathrm{kg}$ of body weight. Titration of $0.01 \mathrm{~N} \mathrm{NaOH}$ was done for estimation of 
acid level using phenolphthalein as an indicator [23]. Level of Pepsin was determined using hemoglobin as a substrate [24].

2.7. Esophageal Myeloperoxidase (MPO) Activity. About $200 \mathrm{mg}$ of esophageal samples were homogenized in $10 \mathrm{vol}-$ umes of cold potassium phosphate buffer $\left(50 \mathrm{mM} \mathrm{K}_{2} \mathrm{HPO}_{4}\right.$, pH 6.0; Sigma, MO, USA) with hexadecyltrimethyl-ammonium bromide (HETAB; 0.5\% w/v; Sigma, MO, USA). After centrifugation at $12,000 \times \mathrm{g}$ and $4^{\circ} \mathrm{C}$ for $10 \mathrm{~min}$, the supernatant was removed and rehomogenization of remains was done with an equivalent volume of $50 \mathrm{mM} \mathrm{K} \mathrm{HPO}_{4}$ containing $0.5 \%(\mathrm{w} / \mathrm{v})$ HETAB and $10 \mathrm{mM}$ EDTA (Sigma, MO, USA). The measurement of $\mathrm{H}_{2} \mathrm{O}_{2}$-dependent oxidation of $o$-dianizidine. $2 \mathrm{HCl}$ was performed for estimation of MPO activity. MPO levels/g of tissue weight that caused a 1.0/min change in absorbance at $460 \mathrm{~nm}$ and $37^{\circ} \mathrm{C}$ was expressed as one unit (U) of enzyme activity [22].

2.8. Measurement of Antioxidative Defense System. Tissue of Fundus was homogenized in cold $0.01 \mathrm{M}$ Tris- $\mathrm{HCl}$ buffer ( $\mathrm{pH}$ 7.4). After centrifugation of homogenate at $800 \times \mathrm{g}$ for $10 \mathrm{~min}$, the supernatant was centrifuged at $12,000 \times \mathrm{g}$ for $15 \mathrm{~min}$ to obtain mitochondrial fraction [25]. Esophageal protein contents were estimated by the Lowry method [26]. Concentration of malondialdehyde (MDA), an indicator of lipid peroxidation (LPO), [27] was determined by the Jamall and Smith's method [28]. The measurement of inhibition of a nicotinamide adenine dinucleotide (reduced)-phenazine methosulphate-nitroblue tetrazolium reaction system was conducted for estimation of superoxide dismutase (SOD) activity [29]. One U of SOD activity, expressed as U/mg protein, is equivalent to $50 \%$ inhibition of the formazan structure for $1 \mathrm{~min}$ at room temperature. The decomposition of $\mathrm{H}_{2} \mathrm{O}_{2}$ in the presence of CAT was measured at $240 \mathrm{~nm}$ [30]. One U of CAT was defined as the amount of enzyme for decomposing $1 \mu \mathrm{mol}$ of $\mathrm{H}_{2} \mathrm{O}_{2} / \mathrm{min}$ at $25^{\circ} \mathrm{C}$ and $\mathrm{pH}$ 7.0. Glutathione (GSH) content was estimated by the method described previously [31].

2.9. Measurement of Gastric Mucosal Component. Total hexose was analyzed by the reaction of carbohydrate in concentrated sulfuric acid with 5-methyl orcinol and measured colorimetrically by previous method [32]. The total hexose was determined according to a standard graph plotted using galactose-mannose. Protein binding of sialic acid was estimated by a thiobarbituric acid assay [33]. After treating esophageal homogenate with $90 \%$ ethanol, the precipitate was dissolved in $0.2 \mathrm{~N}$ sulfuric acid and oxidized with periodic acid and incubated at $37^{\circ} \mathrm{C}$ for $30 \mathrm{~s}$. Oxidation was terminated with sodium arsenate and cyclohexane and $6 \%$ thiobarbituric acid were added. The mixture was centrifuged to obtain a clear pink layer of cyclohexane, and the color intensity was observed at $550 \mathrm{~nm}$. For the measurement of collagen content, the esophageal mucosa was hydrolyzed in $6 \mathrm{~mol} / \mathrm{l} \mathrm{HCl}$ at $110^{\circ} \mathrm{C}$ for $18 \mathrm{~h}$. The evaporations of acid hydrolysates were conducted in a heat block at $95^{\circ} \mathrm{C}$. With $1.0 \mathrm{ml}$ deionized water, the dried residues were rinsed three times with complete evaporation between each wash step. The acid-free samples were suspended again in $1.0 \mathrm{ml}$ acetate-citrate buffer and sonicated for $30 \mathrm{~min}$ [34].

2.10. Blood Histamine Level Detection. The plasma samples were treated with $0.2 \mathrm{M}$ perchloric acid and centrifuged at $10,000 \times \mathrm{g}$ and $4^{\circ} \mathrm{C}$ for $30 \mathrm{~min}$. The supernatant was used for the determination of plasma histamine levels by high performance liquid chromatography [35].

\subsection{Histopathological Processing and Analysis of the Esopha-} gus and Fundus of Stomach. Individual esophagi were fixed in $10 \%$ neutral buffered formalin, and esophageal regions from approximately $5 \mathrm{~cm}$ above the esophageal-cardiac junction and the fundus were prepared for histopathological analysis as described previously [22]. The tissue was trimmed crosswise based on the lumen. All trimmed esophagi and fundi were fixed in 10\% neutral buffered formalin. 3-4 $\mu \mathrm{m}$ sections were prepared after paraffin embedding. Hematoxylin and eosin (H\&E) stain were performed for staining of typical sections and histological profiles were determined. The total thicknesses of esophageal and fundic walls were defined as thickness from luminal mucosal surface to tunica adventitia of esophagus or serosa of fundus. They were measured in the cross-trimmed histological specimens using a digital image analyzer (DMI-300, DMI, Korea). In addition, invasive lesions of esophagus and stomach were estimated using a lesion length and total thickness across the organ wall. Lesion invasiveness (\%) was calculated according to the following equation [22]:

$$
\text { Lesion invasiveness }(\%)=\left(\frac{\text { Length of lesions in the cross-trimmed esophageal or fundic walls }}{\text { total thickness of cross-trimmed esophageal or fundic walls }}\right) \times 100 \text {. }
$$

2.12. Statistical Analyses. Numerical data are presented as means \pm SD According to results of Levene test, when the results showed no significant deviation in variance homogeneity, a one-way analysis of variance (ANOVA) and a least significant difference (LSD) test were done. If there were significant deviations in variance homogeneity, the Kruskal-Wallis $H$ test and Mann-Whitney $U$ (MW) test were conducted. Statistical software was SPSS for Windows release
14.0 K, (SPSS Inc., Chicago, IL, USA), and $P$ value $<0.05$ was considered of statistical significance. In addition, the percent changes compared to sham (PCS) were calculated to estimate the severities of esophageal-fundus damage induced by the RE induction surgery according to (3), and the percent changes compared to control (PCC) were calculated to assess the efficacy of the PD or OMP according to (4): 


$$
\begin{aligned}
& \operatorname{PCS}(\%)=\frac{(\text { Data from control }- \text { Data from sham })}{(\text { Data from sham })} \times 100, \\
& \operatorname{PCC~}(\%)=\frac{(\text { Data from PD or OMP pretreated rats }- \text { Data from control })}{(\text { Data from control })} \times 100 .
\end{aligned}
$$

\section{Results}

3.1. Body Weight Changes and Gross Anatomical Features of the Esophagus and Stomach. There were no differences in body weight among the groups over the course of the 7-day pretreatments (Table 1).

Gross changes were examined in the esophagus and gastric mucosa. Diffused lesions were observed in control compared to sham along with large areas of hemorrhage and ulcer after surgery. However, the size and severity of lesions seemed to be reduced in OMP and all PD groups compared to control (Figure 2).

The mucosal lesion areas were significantly increased in control compared to sham $(P<0.01)$, indicating a suitable induction of RE. The PCS of actual lesion of esophagus and gastric mucosa was $2840.8 \%$ and $1115.3 \%$. Seven-day pretreatment with $\mathrm{PD}$ significantly reduced lesion areas compared to control $(P<0.01)$. The PCC of esophagus lesion areas in PD 200, PD 100, PD 50, and OMP were $-84.9 \%,-55.8 \%$, $-34.8 \%$, and $-54.9 \%$ and those of gastric lesion areas in PD 200, PD 100, PD 50, and OMP were $-73.1 \%,-46.1 \%,-33.4 \%$, and $-42.2 \% \%$, respectively (Figure 3 ).

3.2. Gastric Secretion in the RE Induced Rats. Gastric volume and levels of gastric acid and pepsin were significantly reduced in $\mathrm{PD}$ groups compared to control $(P<0.01)$ (Table 2).

3.3. Analyses of Esophageal Damage. The activity of MPO was significantly increased in control compared with sham $(P<$ 0.01 ) and the PCS of MPO activity was $283.7 \%$, showing granulocyte accumulation after esophageal damage. However, pretreatment with $\mathrm{PD}$ significantly reduced esophageal MPO activity compared to control $(P<0.01)$. The PCC of MPO in PD 200, PD 100, PD 50, and OMP was $-53.3 \%$, $-38.1 \%,-29.4 \%$, and $-36.6 \%$, respectively (Figure 4 ).

3.4. Analyses of Antioxidant Defense Systems. The LPO of control significantly increased compared to sham $(237.2 \%$, $P<0.01)$, reflecting increased cell membrane damage and oxidation by RE induction. LPO was significantly reduced in PD groups compared to control except PD 50. The stomach SOD activity of control was decreased compared to sham $(-53.6 \%, P<0.01)$ and SOD was significantly increased in PD $200(P<0.01)$, PD 100, and PD $50(P<0.05)$ compared to control. CAT of control was reduced compared to sham $(-57.2 \%, P<0.01)$. However, the CAT was significantly increased in PD $200(P<0.01)$ and in PD 100 and PD 50 $(P<0.05)$ compared to control. GSH was reduced in control as compared to sham $(-59.5 \%, P<0.01)$ and significantly increased in PD 200, PD $100(P<0.01)$, and PD $50(P<0.05)$ (Table 3).

3.5. Analyses of Gastric Mucosal Components. The total hexose and sialic acid of control were reduced compared to sham, respectively $(-49.9 \%,-49.5 \%, P<0.01)$. However, they were significantly increased in all PD groups as well as in the OMP group compared to control $(P<0.01)$. PCS of collagen levels was $128.7 \%(P<0.01)$. However, it was significantly reduced in all PD groups compared to control $(P<0.01)$ (Table 4$)$.

3.6. Estimation of Plasma Histamine Levels. Compared to sham, histamine levels appeared to be increased in control (104.7\%, $P<0.01)$. It was significantly decreased in PD 200, PD $100(P<0.01)$, and PD $50(P<0.05)$ compared to control. However, there was no significant changes in OMP as compared to control (Figure 5). The PCC of histamine levels in PD 200, PD 100, PD 50, and OMP were $-35.7 \%,-24.0 \%$, $-12.6 \%$, and $-5.8 \%$, respectively.

3.7. Histopathological Analyses of the Esophagus and Fundus of Stomach. Histopathological assessment showed diffused ulcer and mucosal hyperplasia with hemorrhage and edema in the esophagus and gastric fundus in control (Figure 6(b)). However, these lesions seemed to be milder after the 7-day pretreatment with either PD or OMP as compared to control (Figures 6(c)-6(f)).

Invasive lesion size and total thickness of esophagus were notably increased in control compared to sham $(P<0.01)$. As shown in Table 5, the invasive lesion size was significantly reduced in the PD 200, PD 100, OMP $(P<0.01)$, and PD 50 $(P<0.05)$ compared to control. In addition, total thickness was reduced in all PD groups $(P<0.01)$. Compared to sham, invasive lesion size of fundus of control was increased $(P<$ 0.01). However, the PD and OMP pretreatment groups showed significant reductions in invasive lesions compared to control $(P<0.01)$. Similarly, total thickness was significantly decreased in control compared to sham $(P<0.01)$. The PD 200, PD 100, and OMP pretreatment groups showed a significant increase in the total thickness compared to control $(P<0.01)$ (Table 5).

\section{Discussion}

The Platycodon root is a natural product that has a long history of widespread use in Korean herbal medicine as an antiphlogistic, antitussive, and expectorant agent. The chemical components of platycodin are relatively well-known 


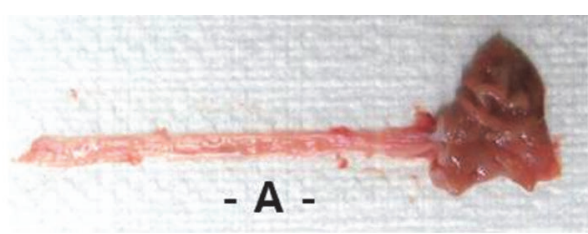

(a)

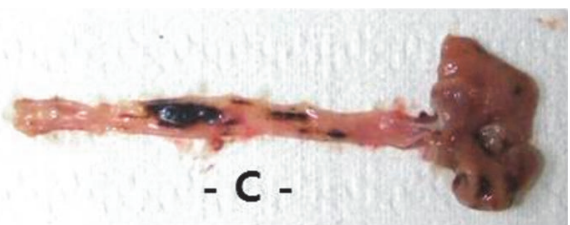

(c)

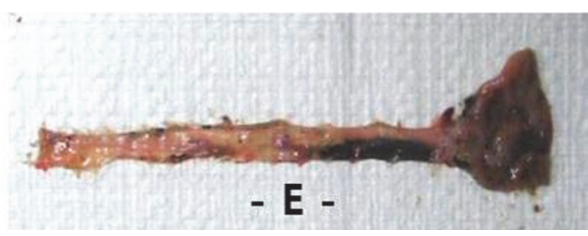

(e)

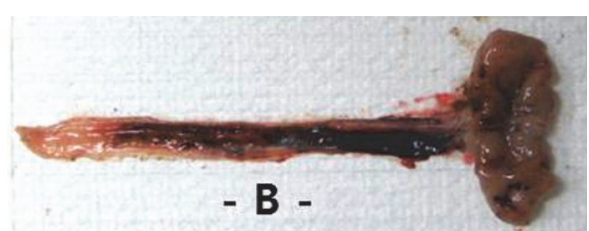

(b)

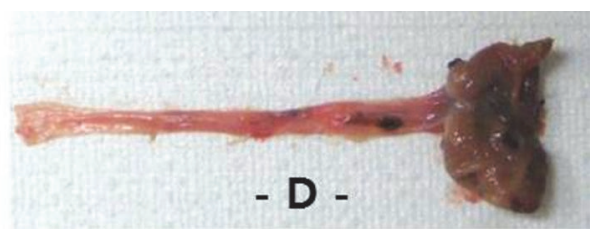

(d)

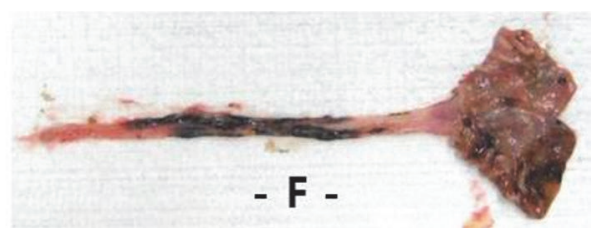

(f)

FIGURE 2: Macroscopic appearance of esophageal and gastric mucosa in pretreatment groups. Panels: (a) sham, (b) control, (c) OMP, (d) PD 200, (e) PD 100, and (f): PD50. Compared to sham (a), severe focal lesions with hemorrhage and ulcer exhibited in the esophageal and gastric mucosa of control (b). However, the macroscopic lesions were dose-dependently reduced by treatment with OMP (c) or PD (d, e, f). PD: platycodin D, OMP: omeprazole.

TABLE 1: Effects of platycodin D on body weight in pretreatment groups.

\begin{tabular}{lccc}
\hline Group & \multicolumn{2}{c}{ Body weight (g) } & \\
& $\begin{array}{c}\text { Day 0 } \\
\text { 1st pretreatment }\end{array}$ & $\begin{array}{c}\text { Day 6 } \\
\text { 7th pretreatment }\end{array}$ & Changes (g) \\
\hline Sham & $176.4 \pm 8.2$ & $190.4 \pm 7.0$ & $14.0 \pm 5.4$ \\
Control & $177.4 \pm 6.1$ & $190.8 \pm 10.6$ & $13.4 \pm 5.6$ \\
OMP & $175.5 \pm 7.5$ & $189.6 \pm 6.4$ & $14.1 \pm 4.6$ \\
PD 200 & $176.3 \pm 9.9$ & $189.5 \pm 11.7$ & $13.3 \pm 6.8$ \\
PD 100 & $176.3 \pm 8.7$ & $190.9 \pm 10.3$ & $14.6 \pm 4.3$ \\
PD 50 & $176.9 \pm 10.5$ & $189.6 \pm 15.7$ & $12.8 \pm 6.5$ \\
\hline
\end{tabular}

The values are expressed as mean $\pm \mathrm{SD}$ of eight rats. Changes were calculated according to (1) in Materials and Methods. PD: platycodin D, OMP: omeprazole.

$[8,9]$, and a single PD compound isolated from the root of Platycodon grandiflorum is available as a commercial dietary supplement for a variety of medicinal applications, such as flu, cough, metabolic disorders, stomachache, diarrhea, and inflammatory infection. In particular, the anti-inflammatory activity of PD has been shown in animals $[13,36]$. In this experiment, 3 different doses of PD were given as pretreatment for 7 days to RE induced rats and the therapeutic effects were compared with a reference drug, OMP, which is one of the most widely used medicines for RE.

Therapeutic Effects of PD on RE. Like the findings of other studies [37-39], PD reduced mucosal inflammation in the



FIGURE 3: Effects of platycodin D on lesion area in the esophageal and gastric mucosa. Values are expressed as mean \pm SD of eight rats; PD: platycodin D, OMP: omeprazole. ${ }^{\#} P<0.01$ compared with sham; ${ }^{*} P<0.01$ compared with control.

esophagus and stomach. The biochemical data revealed reduction of MPO by inhibition of neutrophil infiltration into the injured mucus of the RE, and a reduction of histamine levels by the inhibition of mast cells or basophils in inflammatory pathways (Figures 4 and 5). Although collagen fibers provide structural integrity for tissues under normal conditions, impaired collagen fibers during RE induce sclerosis with stricture formation in the esophagus [40]. Esophageal 
TABLE 2: Effects of platycodin D on gastric secretion.

\begin{tabular}{lccc}
\hline Group & $\begin{array}{c}\text { Gastric volume } \\
(\mathrm{ml} / \mathrm{kg} \text { of body weight })\end{array}$ & $\begin{array}{c}\text { Gastric secretion parameter } \\
\text { Gastric acids } \\
(\mu \mathrm{Eq} / 6 \mathrm{hr})\end{array}$ & $\begin{array}{c}\text { Pepsin } \\
(\mu \mathrm{mol} / 6 \mathrm{hr})\end{array}$ \\
\hline Control & $11.5 \pm 2.1$ & $205.1 \pm 17.1$ & $927.6 \pm 114.6$ \\
OMP & $5.7 \pm 1.0[-50.5]^{*}$ & $95.3 \pm 33.8[-53.6]^{*}$ & $454.3 \pm 211.0[-51.0]^{*}$ \\
PD 200 & $4.5 \pm 1.4[-61.0]^{*}$ & $74.3 \pm 26.8[-63.8]^{*}$ & $312.2 \pm 101.1[-66.4]^{*}$ \\
PD 100 & $5.6 \pm 1.2[-51.3]^{*}$ & $92.3 \pm 25.2[-55.0]^{*}$ & $421.5 \pm 126.4[-54.6]^{*}$ \\
PD 50 & $7.9 \pm 1.7[-31.9]^{*}$ & $123.2 \pm 18.2[-39.9]^{*}$ & $548.5 \pm 102.1[-40.9]^{*}$ \\
\hline
\end{tabular}

Values are expressed as mean \pm SD of eight rats. Data in [ ] mean percent changes compared to control (PCC). ${ }^{*} P<0.01$ compared with control. PD: platycodin D, OMP: omeprazole.

TABLE 3: Antioxidant effects of platycodin D on gastric fundus.

\begin{tabular}{lcccc}
\hline Group & $\begin{array}{c}\text { LPO (nM of MDA/mg } \\
\text { protein) }\end{array}$ & $\begin{array}{c}\text { SOD } \\
\text { (U/mg protein) }\end{array}$ & $\begin{array}{c}\text { Antioxidant defense system } \\
\text { (U/mg protein) }\end{array}$ & $\begin{array}{c}\text { GSH } \\
(\mathrm{mg} / \mathrm{mg} \text { protein) }\end{array}$ \\
\hline Sham & $0.35 \pm 0.17$ & $96.30 \pm 11.01$ & $47.80 \pm 8.51$ & $45.54 \pm 5.34$ \\
Control & $1.17 \pm 0.33^{\# \#}$ & $44.73 \pm 9.93^{\# \#}$ & $20.44 \pm 4.40^{\# \#}$ & $18.46 \pm 1.63^{\# \#}$ \\
OMP & $0.77 \pm 0.20[-34.5]^{\# \#, *}$ & $60.36 \pm 12.10[34.9]^{\# \#, *}$ & $27.75 \pm 3.21[35.8]^{\# \#, *}$ & $25.21 \pm 4.54[36.6]^{\# \#, * *}$ \\
PD 200 & $0.60 \pm 0.23[-48.6]^{\#, * *}$ & $67.70 \pm 19.04[51.4]^{\#, * * *}$ & $31.95 \pm 5.92[56.3]^{\# \#, * *}$ & $28.19 \pm 5.19[52.7]^{\# \#, * *}$ \\
PD 100 & $0.74 \pm 0.14[-36.6]^{\# \#, * *}$ & $60.31 \pm 9.98[34.8]^{\#, *}$ & $27.84 \pm 4.69[36.2]^{\#, *}$ & $24.45 \pm 4.21[34.1]^{\# \#, * *}$ \\
PD 50 & $0.87 \pm 0.13[-25.8]^{\# \#}$ & $55.58 \pm 7.53[24.3]^{\# \#, *}$ & $26.07 \pm 4.65[27.5]^{\# \#, *}$ & $23.40 \pm 3.70[26.8]^{\# \#, *}$ \\
\hline
\end{tabular}

Values are expressed as mean \pm SD of eight rats; LPO: lipid peroxidation; MDA: malondialdehyde; SOD: superoxide dismutase; CAT: catalase; GSH: glutathione. Data in [ ] mean percent changes compared to control (PCC). PD: platycodin D, OMP: omeprazole. ${ }^{\#} P<0.05$ and ${ }^{\# \#} P<0.01$ compared with sham; ${ }^{*} P<0.05$ and ${ }^{* *} P<0.01$ compared with control.

TABLE 4: Effects of platycodin D on gastric mucosal components.

\begin{tabular}{lccc}
\hline Group & $\begin{array}{c}\text { Total hexose } \\
(\mu \mathrm{g} / 100 \mathrm{mg} \text { tissue })\end{array}$ & $\begin{array}{c}\text { Gastric mucosal component } \\
\text { Sialic acid } \\
(\mu \mathrm{g} / 100 \mathrm{mg} \text { tissue })\end{array}$ & $\begin{array}{c}\text { Collagen } \\
(\mathrm{mg} / 100 \mathrm{mg} \text { tissue })\end{array}$ \\
\hline Sham & $2675.67 \pm 500.42$ & $149.23 \pm 15.16$ & $18.72 \pm 2.45$ \\
Control & $1340.48 \pm 202.74^{\#}$ & $75.44 \pm 16.03^{\#}$ & $42.81 \pm 5.93^{\#}$ \\
OMP & $1946.79 \pm 179.24[45.2]^{\#, *}$ & $109.91 \pm 12.88[45.7]^{\#, *}$ & $28.08 \pm 5.66[-34.4]^{\#, *}$ \\
PD 200 & $2206.21 \pm 350.50[64.6]^{*}$ & $123.72 \pm 10.50[64.0]^{\#, *}$ & $21.41 \pm 3.36[-50.0]^{*}$ \\
PD 100 & $1943.86 \pm 232.70[45.0]^{\#, *}$ & $108.28 \pm 12.00[43.5]^{\#, *}$ & $28.52 \pm 4.50[-33.4]^{\#, *}$ \\
PD 50 & $1811.29 \pm 235.21[35.1]^{\#, *}$ & $101.15 \pm 7.91[34.1]^{\#, *}$ & $30.99 \pm 4.76[-27.6]^{\#, *}$ \\
\hline
\end{tabular}

Values are expressed as mean \pm SD of eight rats. Data in [ ] mean percent changes compared to control (PCC). PD: platycodin D, OMP: omeprazole. ${ }^{\#} P<0.01$ compared with sham; ${ }^{*} P<0.01$ compared with control.

TABLE 5: Therapeutic effects of platycodin D on esophagus and fundus of stomach.

\begin{tabular}{lcccc}
\hline \multirow{2}{*}{ Group } & \multicolumn{2}{c}{ Esophagus } & \multicolumn{2}{c}{ Fundic stomach } \\
& Invasive lesion (\%) & Thickness $(\mu \mathrm{m})$ & Invasive lesion $(\%)$ & Thickness $(\mu \mathrm{m})$ \\
\hline Sham & $1.42 \pm 0.95$ & $649.18 \pm 139.75$ & $3.87 \pm 3.83$ & $1802.52 \pm 255.56$ \\
Control & $83.62 \pm 8.24^{\#}$ & $1785.47 \pm 269.89^{\#}$ & $62.66 \pm 7.31^{\#}$ & $946.72 \pm 161.64^{\#}$ \\
OMP & $54.30 \pm 8.41[-35.1]^{\#, * *}$ & $939.09 \pm 155.01[-45.0]^{\#, * *}$ & $30.14 \pm 3.70[-51.9]^{\#, * *}$ & $1362.21 \pm 273.10[43.4]^{\#, * *}$ \\
PD 200 & $40.73 \pm 4.63[-51.3]^{\#, * *}$ & $857.87 \pm 189.94[-52.0]^{* * *}$ & $12.55 \pm 5.04[-80.0]^{\#, * *}$ & $1554.51 \pm 321.09[63.7]^{* *}$ \\
PD 100 & $53.86 \pm 9.93[-35.6]^{\#, * *}$ & $988.39 \pm 186.60[-44.6]^{\#, * *}$ & $31.27 \pm 8.27[-50.1]^{\#, * *}$ & $1361.31 \pm 300.32[43.3]^{\#, * *}$ \\
PD 50 & $68.03 \pm 10.21[-18.6]^{\#, *}$ & $1189.89 \pm 266.96[-33.3]^{\#, * *}$ & $46.77 \pm 6.91[-25.4]^{\#, * *}$ & $1156.39 \pm 114.53[21.8]^{\#}$ \\
\hline
\end{tabular}

Values are expressed as mean \pm SD of eight rats. The percentage of invasive lesions and total thickness through the esophageal or gastric wall was measured using a microscopic analyzer. Data in [ ] mean percent changes compared to control. PD: platycodin D, OMP: omeprazole. ${ }^{\#} P<0.01$ compared with sham; ${ }^{*} P<0.05$ and ${ }^{* *} P<0.01$ compared with control. 




FIGURE 4: Effects of platycodin D on myeloperoxidase activity. Values are expressed as mean \pm SD of eight rats; ${ }^{\#} P<0.01$ compared with sham; ${ }^{*} P<0.01$ compared with control. PD: platycodin $\mathrm{D}$, OMP: omeprazole.

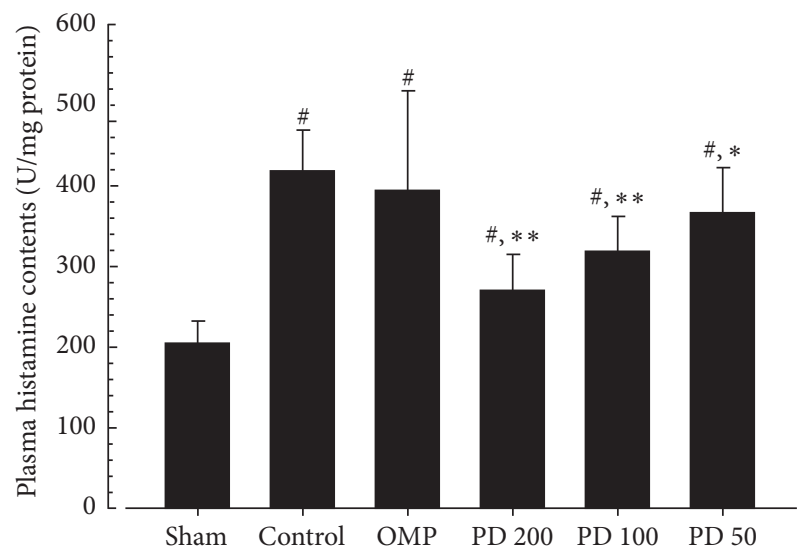

Figure 5: Effects of platycodin D on levels of plasma histamine. Values are expressed as mean \pm SD of eight rats. PD: platycodin $\mathrm{D}$, OMP: omeprazole. ${ }^{\#} P<0.01$ compared with sham; ${ }^{*} P<0.05$ and ${ }^{* *} P<0.01$ compared with control.

collagen levels were reduced in PD groups as well as in the OMP group compared to control (Table 4). The antiinflammatory effects of PD resulted in an improvement in the lesions associated with mucosal injuries, showing a reduction in gross lesion area (Figures 2 and 3) and gastric contents including acids and pepsin (Table 2). PD treatment was also associated with increases in the levels of gastric mucosa components, total hexose and sialic acids (Table 4), and milder histopathological lesions (Table 5 and Figure 6). Gastric contents, including acids and pepsin secreted from parietal cells, are a potentially damaging factor in the esophageal and gastric mucosa. The reduced histamine levels may inhibit the secretion of gastric acids from the parietal cells that normally occurs in response to histamine and reduce damage to the mucosal membranes [41, 42]. Therefore, these findings suggest that PD has therapeutic potential for gastroesophageal reflux disease.
Antioxidative Effects of PD on RE. The pathogenesis of RE has multiple factors, particularly since the gastric contents refluxing into the esophagus contain a variety of components $[43,44]$. Although exposure of the esophageal membranes to gastric acids influences the severity of RE, oxidative stress also plays an important role in mucosal erosion as well as the secondary damage to the mucosal layer by mechanical digestive movement [6]. In present study, LPO, SOD, CAT, and GSH were used to prove for antioxidative effects of PD. Increased production of oxygen-derived free radicals is accompanied by enhanced mucosal lipid peroxidation, which is used as an indicator of mucosal membrane damage caused by free radicals [5]. The control showed highly increased LPO (3.4-fold) as compared to sham. However, LPO was significantly reduced in the PD 200 and PD 100 pretreatment groups (Table 3 ), suggesting inhibition of oxygen-derived free radical production by treatment with higher doses of $\mathrm{PD}$. This suggests that PD inhibits oxidative stress by enhancing antioxidant enzymes, which is supported by the increased levels of SOD, CAT, and GSH in all PD groups (Table 3 ). Catalase (CAT) activity is represented for capacity to degrade hydrogen peroxide which produces hypochlorous acid and the highly toxic hydroxyl free radical [45]. Glutathione (GSH) is involved in preventing oxygen-derived free radicals from contributing to the pathogenesis of RE $[46,47]$.

Relevance of Antioxidant Effects for RE Therapeutics. Since the esophageal and gastric mucosa are injured not only by gastric acids alone but also by oxidative stress and oxygen-derived free radicals, therapeutic strategies also focus on antioxidants as well as regulating $\mathrm{pH}$. One approach may involve enhancement of the natural antioxidative mechanisms in the body, for example, upregulation of antioxidative enzymes such as SOD, CAT, or GSH. There has been extensive research into natural herbal-derived compounds for combating oxidative stress and free radical damage [48]. A 7-day pretreatment with $\mathrm{PD}$ enhanced antioxidative pathways in RE induced rats suggests that PD can function as a free radical scavenger that can oppose oxidative stress. Another approach is treatment with exogenous antioxidants. Thiol-containing $\mathrm{GSH}$, a nonenzyme antioxidant, promotes the detoxification of several toxic metabolites and exogenous thiol compounds have been shown to protect the stomach from ethanolinduced injuries [47]. An acidic environment and histamine release can both induce degradation of mucosal glycoprotein $[46,49]$. In addition, high levels of histamine secretion have been suggested to be involved in the pathogenesis of gastric ulceration, inflammation, esophagitis, and gastric acid secretion [42]. The antioxidant effects of PD may be involved in the observed reduction in histamine levels, leading to the increased levels of glycoproteins (Table 4). Indeed, the histopathological lesions associated with the experimental RE used here appeared to be ameliorated by PD treatment (Figure 6). OMP showed similar effects with PD except on histamine levels, which is in agreement with other studies $[23,50]$. It is probably due to the function of OMP as a PPI involved in reducing gastric acids by inhibition of proton pump rather than inhibiting oxidative stress. Therefore, $\mathrm{PD}$ may have a therapeutic efficacy similar to or better than OMP in human patients with RE. 


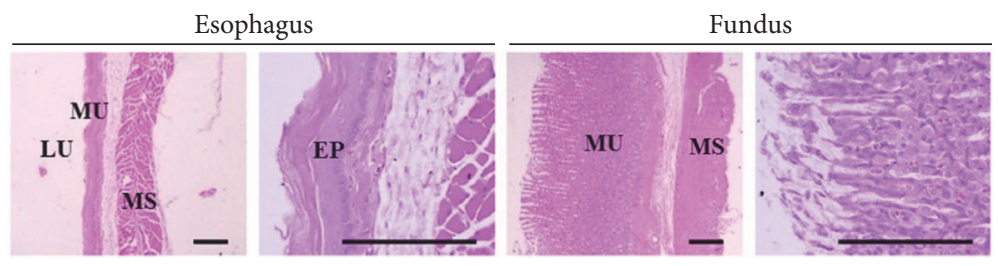

(a)

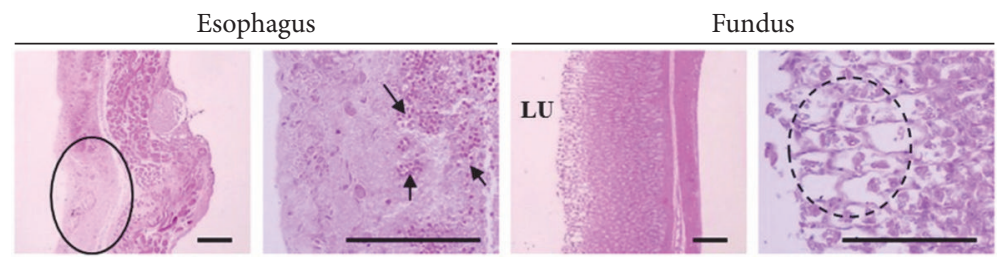

(b)

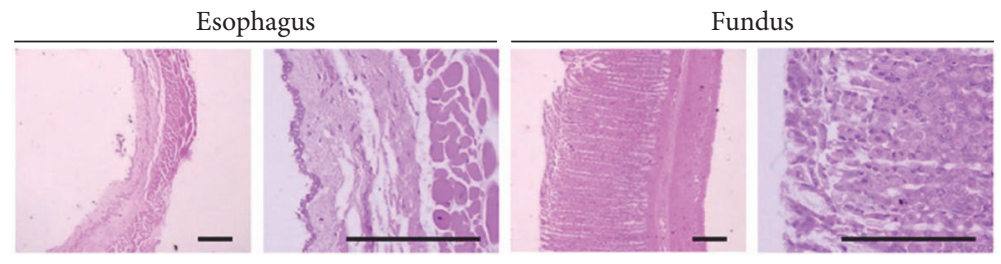

(c)


(d)

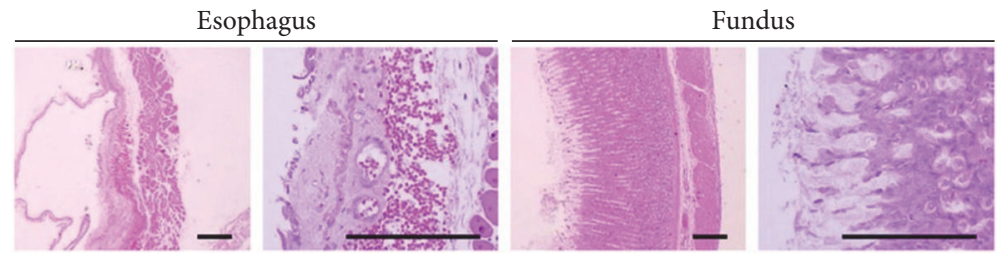

(e)
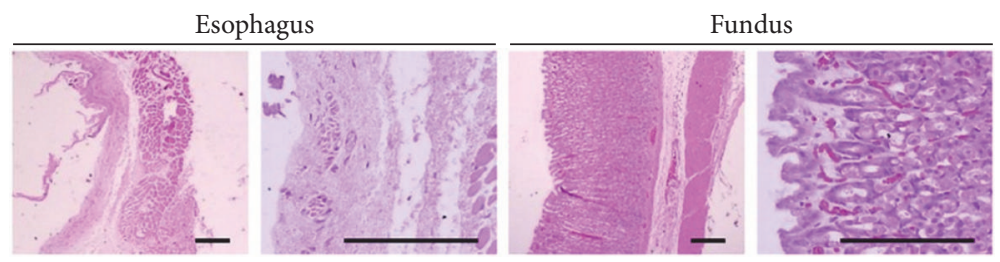

(f)

FIGURE 6: Histopathological analyses of invasive lesions and total organ wall thickness. The panels show representative histopathological profiles of a hematoxylin-eosin stain of the esophagus and gastric fundus in sham or RE induced rats. Panels: (a) sham, (b) control, (c) OMP, (d) PD 200, (e) PD 100, and (f) PD50. Histopathological analysis showed severe focal lesions with hemorrhage (black arrow), ulcer (circle), and edematous changes (dashed line circle) in the esophagus and gastric fundus of control compared with sham. However, the lesions were notably reduced by treatment with each of the 3 doses of PD compared with control; PD: platycodin D; OMP: omeprazole; LU: lumen; EP: epithelium; MU: mucosa; MS: muscular layer; Scale bars $=80 \mu \mathrm{m}$. 
Other Mechanisms Involved in the Therapeutic Effects of PD. The therapeutic effects of PD on RE may result from immunomodulatory properties involving secretion of chemical mediators and migration of inflammatory cells. PD has been shown to suppress NO secretion and increase TNF- $\alpha$ in macrophages activated by lipopolysaccharide and interferon(IFN-) $\gamma$ [20]. In addition, PD suppresses the secretion of prostaglandin E2 (PGE2) in rat peritoneal macrophages stimulated by a protein kinase $\mathrm{C}$ activator $[13,20]$. Seven-day pretreatment with PD showed significant inhibition of inflammation with a reduction in vascular permeability, leukocyte migration, and edema in RE model rats. In addition, antioxidant effects of PD were observed by a reduction in LPO and increases in the antioxidant enzymes SOD, CAT, and GSH. This suggests that PD downregulates NO synthesis, which may inhibit the production of free radicals, resulting in improvement of RE.

Novel Drug Development Using Natural Herbal Products. Currently, there has been an upsurge in research into developing novel drugs using herbal products [51]. PD contains five sugars identified as derivatives of oleanolic acid, a fatty acid. The number and types of sugar residues are thought to be involved in the diverse pharmacologic effects of PD. PD 3 is another single compound derived from Platycodon roots that is composed of six sugars and has less inhibitory effects on NO production and less stimulatory effects on TNF activity. In addition, while PD suppresses PGE2 production, PD 3 does not [13]. Another example is ginseng, which is composed of panaxadiol and panaxatriol with more than 10 derivatives [52]. Depending on the number of sugars, ginseng can have a variety of effects including antioxidant effects, anticancer properties, or immunomodulatory function [53]. Currently flourishing research has explored their therapeutic effects, and the chemical structures of novel drugs have been directly impacted by research into natural products. Therefore, further studies of pharmacological activity based on the structure of single compounds isolated from effective natural herbal products may provide important information for novel drug discovery.

\section{Conclusion}

The study revealed the therapeutic potential of PD regarding the suppression of histamine and gastric acid secretion accompanied by an enhancement of antioxidative system function in the gastrointestinal tract. PD has been used as a dietary supplement or medication in Korea with low potential for toxicity in humans, so further insights are expected from clinical trials designed to compare the efficacy of PD with other chemical compounds. This study provided important information, not only for RE therapeutics but also for drug discovery based on the structures of herbal compounds.

\section{Disclosure}

This has been published partially as the form of an abstract for poster session at a congress of the European Society of Toxicology (Toxicology Letters, 229S, 2014).

\section{Conflicts of Interest}

The authors declare that there are no conflicts of interest regarding the publication of this paper.

\section{Authors' Contributions}

Su-Yeon Cho and Chang-Hyun Song have contributed equally to the research and preparation of this manuscript.

\section{Acknowledgments}

This work was supported by the National Research Foundation of Korea (NRF) grant funded by the Korea government (MSIP) (no. 2012R1A5A2A42671316).

\section{References}

[1] A. Kandulski and P. Malfertheiner, "Gastroesophageal reflux disease-from reflux episodes to mucosal inflammation," Nature Reviews Gastroenterology \& Hepatology, vol. 9, no. 1, pp. 1522, 2012.

[2] M. B. Fennerty, "The continuum of GERD complications," Cleveland Clinic Journal of Medicine, vol. 70, no. supplement 5, pp. S33-S33, 2003

[3] J. Dent, H. B. El-Serag, M.-A. Wallander, and S. Johansson, "Epidemiology of gastro-oesophageal reflux disease: a systematic review," Gut, vol. 54, no. 5, pp. 710-717, 2005.

[4] H. S. Kim, S. J. Baik, K. H. Kim et al., "Prevalence of and risk factors for gastrointestinal diseases in korean americans and native koreans undergoing screening endoscopy," Gut and Liver, vol. 7, no. 5, pp. 539-545, 2013.

[5] A. Farhadi, J. Fields, A. Banan, and A. Keshavarzian, "Reactive oxygen species: are they involved in the pathogenesis of GERD, Barrett's esophagus, and the latter's progression toward esophageal cancer?" American Journal of Gastroenterology, vol. 97, no. 1, pp. 22-26, 2002.

[6] T. Y. Oh, J. S. Lee, B. O. Ahn et al., "Oxidative stress is more important than acid in the pathogenesis of reflux oesophagitis in rats," Gut, vol. 49, no. 3, pp. 364-371, 2001.

[7] K. R. Devault and D. O. Castell, "Updated guidelines for the diagnosis and treatment of gastroesophageal reflux disease," American Journal of Gastroenterology, vol. 94, no. 6, pp. 1434$1442,1999$.

[8] A. Tada, Y. Kaneiwa, J. Shoji, and S. Shibata, "Studies on the saponins of the root of Platycodon grandiflorum A. De Candolle. I. Isolation and the structure of platycodin-D," Chemical \& Pharmaceutical Bulletin, vol. 23, no. 11, pp. 2965-2972, 1975.

[9] T. Saeki and T. Nikaido, "Evaluations of saponin properties of HPLC analysis of Platycodon grandiflorum A.DC.," Yakugaku Zasshi, vol. 123, no. 6, pp. 431-441, 2003.

[10] W. Li, Y.-H. Tian, Y. Liu, Z. Wang, S. Tang, and J. Zhang, "Platycodin D exerts anti-tumor efficacy in $\mathrm{H}_{22}$ tumor-bearing mice via improving immune function and inducing apoptosis," Journal of Toxicological Sciences, vol. 41, no. 3, pp. 417-428, 2016.

[11] Y. Kong, Z.-L. Lu, J.-J. Wang et al., "Platycodin D, a metabolite of Platycodin grandiflorum, inhibits highly metastatic MDA-MB231 breast cancer growth in vitro and in vivo by targeting the MDM2 oncogene," Oncology Reports, vol. 36, no. 3, pp. 14471456, 2016. 
[12] J. W. Chung, E. J. Noh, H. L. Zhao et al., "Anti-inflammatory activity of prosapogenin methyl ester of platycodin D via nuclear factor-kappaB pathway inhibition," Biological \& Pharmaceutical Bulletin, vol. 31, no. 11, pp. 2114-2120, 2008.

[13] Y. P. Kim, E. B. Lee, S. Y. Kim et al., "Inhibition of prostaglandin E2 production by platycodin D isolated from the root of Platycodon grandiflorum," Planta Medica, vol. 67, no. 4, pp. 362-364, 2001.

[14] E. J. Lee, M. Kang, and Y. S. Kim, "Platycodin D inhibits lipogenesis through AMPK $\alpha$-PPAR $\gamma 2$ in 3T3-L1 cells and modulates fat accumulation in obese mice," Planta Medica, vol. 78, no. 14, pp. 1536-1542, 2012.

[15] Y.-M. Ahn, S. K. Kim, J.-S. Kang, and B.-C. Lee, "Platycodon grandiflorum modifies adipokines and the glucose uptake in high-fat diet in mice and L6 muscle cells," Journal of Pharmacy and Pharmacology, vol. 64, no. 5, pp. 697-704, 2012.

[16] H. L. Zhao, K.-H. Cho, Y. W. Ha, T.-S. Jeong, W. S. Lee, and Y. S. Kim, "Cholesterol-lowering effect of platycodin D in hypercholesterolemic ICR mice," European Journal of Pharmacology, vol. 537, no. 1-3, pp. 166-173, 2006.

[17] S.-S. Choi, E.-J. Han, T.-H. Lee, K.-J. Han, H.-K. Lee, and H.W. Suh, "Antinociceptive profiles of platycodin D in the mouse," American Journal of Chinese Medicine, vol. 32, no. 2, pp. 257268, 2004.

[18] Y. Xie, W. Deng, H. Sun, and D. Li, "Platycodin D2 is a potential less hemolytic saponin adjuvant eliciting Th1 and Th2 immune responses," International Immunopharmacology, vol. 8, no. 8, pp. 1143-1150, 2008.

[19] J. H. Choi, K.-Y. Yoo, O. K. Park et al., "Platycodin D and 2" -oacetyl-polygalacin D2 isolated from Platycodon grandiflorum protect ischemia/reperfusion injury in the gerbil hippocampus," Brain Research, vol. 1279, pp. 197-208, 2009.

[20] C. Wang, G. B. Schuller Levis, E. B. Lee et al., "Platycodin D and D3 isolated from the root of Platycodon grandiflorum modulate the production of nitric oxide and secretion of TNF- $\alpha$ in activated RAW 264.7 cells," International Immunopharmacology, vol. 4, no. 8, pp. 1039-1049, 2004.

[21] F. Massoomi, J. Savage, and C. J. Destache, "Omeprazole: a comprehensive review," Pharmacotherapy, vol. 13, no. 1, pp. 46-59, 1993.

[22] S.-K. Ku, B.-I. Seo, and J.-H. Park, "Effect of Lonicerae Flos extracts on reflux esophagitis with antioxidant activity," World Journal of Gastroenterology, vol. 15, no. 38, pp. 4799-4805, 2009.

[23] C. V. Rao and M. Vijayakumar, "Effect of quercetin, flavonoids and $\alpha$-tocopherol, an antioxidant vitamin on experimental reflux oesophagitis in rats," European Journal of Pharmacology, vol. 589, no. 1-3, pp. 233-238, 2008.

[24] K. Sairam, C. V. Rao, M. D. Babu, K. V. Kumar, V. K. Agrawal, and R. K. Goel, "Antiulcerogenic effect of methanolic extract of Emblica officinalis: an experimental study," Journal of Ethnopharmacology, vol. 82, no. 1, pp. 1-9, 2002.

[25] C. V. Rao, S. K. Ojha, K. Radhakrishnan et al., "Antiulcer activity of Utleria salicifolia rhizome extract," Journal of Ethnopharmacology, vol. 91, no. 2-3, pp. 243-249, 2004.

[26] O. H. Lowry, N. J. Rosebrough, A. L. Farr, and R. J. Randall, "Protein measurement with the Folin phenol reagent," The Journal of Biological Chemistry, vol. 193, no. 1, pp. 265-275, 1951.

[27] M. Masayasu and Y. Hiroshi, "A simplified assay method of superoxide dismutase activity for clinical use," Clinica Chimica Acta, vol. 92, no. 3, pp. 337-342, 1979.
[28] I. S. Jamall and J. C. Smith, "Effects of cadmium on glutathione peroxidase, superoxide dismutase, and lipid peroxidation in the rat heart: a possible mechanism of cadmium cardiotoxicity," Toxicology and Applied Pharmacology, vol. 80, no. 1, pp. 33-42, 1985.

[29] P. Kakkar, B. Das, and P. N. Viswanathan, "A modified spectrophotometric assay of superoxide dismutase," Indian Journal of Biochemistry \& Biophysics, vol. 21, no. 2, pp. 130-132, 1984.

[30] H. Aebi, "Methods in enzymatic analysis", in Catalase, H. U. Bergmeyer, Ed., pp. 673-686, Academic Press Inc, New York, NY, USA, 1974.

[31] M. S. Moron, J. W. Depierre, and B. Mannervik, "Levels of glutathione, glutathione reductase and glutathione S-transferase activities in rat lung and liver," Biochimica et Biophysica Acta, vol. 582, no. 1, pp. 67-78, 1979.

[32] R. J. Winzler, Methods of Biochemical Analysis, Interscience Publishers Inc, New York, NY, USA, 1955.

[33] L. Warren, "The thiobarbituric acid assay of sialic acids," The Journal of Biological Chemistry, vol. 234, no. 8, pp. 1971-1975, 1959.

[34] J. F. Woessner Jr., “The determination of hydroxyproline in tissue and protein samples containing small proportions of this imino acid," Archives of Biochemistry and Biophysics, vol. 93, no. 2, pp. 440-447, 1961.

[35] Y. Tsuruta, K. Kohashi, and Y. Ohkura, "Simultaneous determination of histamine and $\mathrm{N} \tau$-methylhistamine in human urine and rat brain by high-performance liquid chromatography with fluorescence detection," Journal of Chromatography B: Biomedical Sciences and Applications, vol. 224, no. 1, pp. 105-110, 1981.

[36] T. Zhang, S. Yang, J. Du, Y. Jinfu, and W. Shumin, "Platycodin $\mathrm{D}$ attenuates airway inflammation in a mouse model of allergic asthma by regulation NF- $\kappa \mathrm{B}$ pathway," Inflammation, vol. 38, no. 3, pp. 1221-1228, 2015.

[37] J. C. Chen, L. J. Li, S. M. Wen, Y. C. He, H. X. Liu, and Q. S. Zheng, "Quantitative analysis and simulation of anti-inflammatory effects from the active components of Paino Powder in rats," Chinese Journal of Integrative Medicine, 2011.

[38] K. S. Ahn, E. J. Noh, H. L. Zhao, S. H. Jung, S. S. Kang, and Y. S. Kim, "Inhibition of inducible nitric oxide synthase and cyclooxygenase II by Platycodon grandiflorum saponins via suppression of nuclear factor- $\kappa \mathrm{B}$ activation in RAW 264.7 cells," Life Sciences, vol. 76, no. 20, pp. 2315-2328, 2005.

[39] Y. S. Chan, J. L. Woo, B. L. Eun, Y. C. Eui, and H. K. Kwang, "Platycodin D and D3 increase airway mucin release in vivo and in vitro in rats and hamsters," Planta Medica, vol. 68, no. 3, pp. 221-225, 2002.

[40] L. Hendel, "Hydroxyproline in the oesophageal mucosa of patients with progressive systemic sclerosis during omeprazoleinduced healing of reflux oesophagitis," Alimentary Pharmacology \& Therapeutics, vol. 5, no. 5, pp. 471-480, 1991.

[41] T. Brzozowski, P. C. Konturek, S. J. Konturek et al., "Role of gastric acid secretion in progression of acute gastric erosions induced by ischemia-reperfusion into gastric ulcers," European Journal of Pharmacology, vol. 398, no. 1, pp. 147-158, 2000.

[42] M. Kitano, M. Bernsand, Y. Kishimoto et al., "Ischemia of rat stomach mobilizes ECL cell histamine," American Journal of Physiology-Gastrointestinal and Liver Physiology, vol. 288, no. 5, pp. G1084-G1090, 2005.

[43] P. Malfertheiner and B. Hallerbäck, "Clinical manifestations and complications of gastroesophageal reflux disease (GERD)," 
International Journal of Clinical Practice, vol. 59, no. 3, pp. 346355, 2005.

[44] R. D. S. Pereira, "Regression of gastroesophageal reflux disease symptoms using dietary supplementation with melatonin, vitamins and aminoacids: Comparison with omeprazole," Journal of Pineal Research, vol. 41, no. 3, pp. 195-200, 2006.

[45] B. Halliwell, "Free radicals, antioxidants, and human disease: curiosity, cause, or consequence?” The Lancet, vol. 344, no. 8924, pp. 721-724, 1994.

[46] S. K. Ahlawat, R. Mohi-Ud-Din, D. C. Williams, K. A. Maher, and S. B. Benjamin, "A prospective study of gastric acid analysis and esophageal acid exposure in patients with gastroesophageal reflux refractory to medical therapy," Digestive Diseases and Sciences, vol. 50, no. 11, pp. 2019-2024, 2005.

[47] A. Robert, D. Eberle, and N. Kaplowitz, "Role of glutathione in gastric mucosal cytoprotection," American Journal of Physiology-Gastrointestinal and Liver Physiology, vol. 247, no. 3, part 1, pp. G296-G304, 1984.

[48] H. Wiseman and B. Halliwell, "Damage to DNA by reactive oxygen and nitrogen species: role in inflammatory disease and progression to cancer," Biochemical Journal, vol. 313, no. 1, pp. 17-29, 1996.

[49] A. Allen, G. Flemstrom, A. Garner, and E. Kivilaakso, "Gastroduodenal mucosal protection," Physiological Reviews, vol. 73, no. 4, pp. 823-857, 1993.

[50] Ü. Biçakçi, B. Tander, E. Aritürk et al., "Effects of omeprazole and gentamicin on the biochemical and histopathological alterations of the hypoxia/reoxygenation induced intestinal injury in newborn rats," Pediatric Surgery International, vol. 21, no. 10, pp. 800-805, 2005.

[51] H.-F. Ji, X.-J. Li, and H.-Y. Zhang, "Natural products and drug discovery," EMBO Reports, vol. 10, no. 3, pp. 194-200, 2009.

[52] M. R. Harkey, G. L. Henderson, M. E. Gershwin, J. S. Stern, and R. M. Hackman, "Variability in commercial ginseng products: an analysis of 25 preparations," American Journal of Clinical Nutrition, vol. 73, no. 6, pp. 1101-1106, 2001.

[53] D. Kiefer and T. Pantuso, "Panax ginseng," American Family Physician, vol. 68, no. 8, pp. 1539-1542, 2003. 